Relations industrielles

Industrial Relations

\title{
The Motivation to work, by F. Herzberg, B. Mausner and B.-C. Snyderman, John Wiley \& Sons, New York, John Wiley \& Sons, 1959.
}

\section{C.-R. Giroux}

Volume 15, numéro 2, avril 1960

URI : https://id.erudit.org/iderudit/1022040ar

DOI : https://doi.org/10.7202/1022040ar

Aller au sommaire du numéro

Éditeur(s)

Département des relations industrielles de l’Université Laval

ISSN

0034-379X (imprimé)

1703-8138 (numérique)

Découvrir la revue

Citer ce compte rendu

Giroux, C.-R. (1960). Compte rendu de [The Motivation to work, by F. Herzberg, B. Mausner and B.-C. Snyderman, John Wiley \& Sons, New York, John Wiley \& Sons, 1959.] Relations industrielles / Industrial Relations, 15(2), 275-276.

https://doi.org/10.7202/1022040ar

Tous droits réservés @ C Département des relations industrielles de l’Université Laval, 1960
Ce document est protégé par la loi sur le droit d'auteur. L'utilisation des services d'Érudit (y compris la reproduction) est assujettie à sa politique d'utilisation que vous pouvez consulter en ligne.

https://apropos.erudit.org/fr/usagers/politique-dutilisation/ 
absolument indispensables si nous voulons atteindre les buts proposés par Macdonald et ses successeurs.

La radio et la télévision privées, même avec la meilleure réglementation possible, ne peuvent tout simplement pas remplir cette tâche. Leur premier objectif est de faire de l'argent et non pas de bâtir une nation. C'est naturel, inévitable et normal. Ils peuvent et doivent rendre des services publics mais ce sont les bénéfices qui demeurent leur objectif principal. Les bénéfices, même avec les plus sévères des règlements, nous attireront dans l'orbite des Etats-Unis, des programmes américains ou du genre américain. Pour bâtir notre pays, nous avons besoin de Radio-Canada et Radio-Canada est le genre d'institution que ses fondateurs et les gouvernements successifs ont toujours envisagée.

Cela va nous coûter de l'argent comme le C.P.R. et la Politique nationale nous en ont coûté; avoir un Canada bien à nous coûte de l'argent. Si nous voulons avoir une véritable nation canadienne, nous devons être prêts à en payer le prix. Une partie de ce prix consistera à fournir des fonds importants à Radio-Canada. Faire des économies avec Radio-Canada, c'est faire des économies avec notre existence nationale.

\section{RECENSIONS - BOOK REVIEWS}

The Motivation to work, by F. Herzberg, B. Mausner and B.-C. Snyderman, John Wiley \& Sons, New York, 1959.

Après avoir résumé et évalué les divers articles et différentes recherches sur les attitudes des employés envers leur travail (Job Attitudes: Review of Research and Opinions), il était tout naturel pour ces chercheurs d'essayer de prouver quelques-unes des hypothèses émises dans leurs conclusions.

$\mathrm{Au}$ nombre de ces hypothèses deux ont surtout attiré leur attention. D'abord, ils ont prétendu que la satisfaction envers le travail ne s'étendait pas sur un seul continuum allant d'extrêmement satisfait à extrêmement mécontent, mais qu'il y avait un continuum de satisfaction et un autre de mécontentement. L'échantillonnage de leur recherche comprend des ingénieurs et des comptables.

Le but de leur recherche est d'approfondir et de comprendre le syndrome du moral qui progresse selon la séquence suivante: situation - attitudes - effets.
Ce phénomène complexe peut être analysé de deux façons: l'approche «nomothéthique » i.e. statistique et l'approche «idiographique » i.e. entrevue individuelle.

La première méthode prétend qu'il est possible de délimiter des groupes d'employés dont le niveau de moral est différent: moral élevé et moral bas. Ensuite, ces groupes sont comparés entre eux en fonction d'autres variables. L'or. calcule des indices de corrélations entre le moral et diverses mesures de performance. L'approche «idiographique » prétend que le complexe \& facteurs attitudes - effets 》 doit être étudié directement chez l'individu. En d'autres termes, il faut essayer de comprendre ce que provoque une situation particulière d'un individu à l'autre.

Les auteurs ont éliminé l'approche statistique, car elle est contaminée de plusieurs façons. Ils utilisèrent la méthode d'entrevue individuelle pour obtenir des données pour leur recherche. L'investigateur posait les questions suivantes à la personne interviewée: \& Pensez à une époque où vous étiez excep- 
tionnellement bien disposé ou mal disposé envers votre travail. Quel fait a provoqué cette situation? Qu'elle a été votre attitude par la suite? Quel effet votre attitude a-t-elle eu sur votre travail ? Les entrevues furent analysées selon la méthode d'analyse du contenu de Berelson. Le contenu de l'entrevue est séparé en catégories. Toutefois cette distribution permet de conserver la séquence ou mieux le lien individuel entre les facteurs, les attitudes et les effets.

Les résultats de cette analyse sont des plus révélateurs et des plus lumineux.

Ils viennent confirmer les hypothèses émises au début de l'ouvrage. Un groupe de facteurs se rapportent au continuum de satisfaction: reconnaissance, responsabilité, possibilité de croissance, succès au travail, le contenu du travail. Lorsque ces facteurs produisent un sentiment de satisfaction, ceci a pour effet de maintenir l'individu dans un état d'équilibre ou d'augmenter sa motivation et en même temps d'améliorer sa performance: il est très important de remarquer que ces facteurs relèvent du contenu même de la fonction de l'individu.

Le deuxième groupe de facteurs continuum de mécontentement contribue surtout à la dissatisfaction des employés: politique de compagnie, administration, qualité de la supervision, salaire, relations interpersonnelles, conditions de travail. Il est à remarquer que tous ces facteurs s'apparentent aux conditions de travail, ils sont concomitants à la fonction elle-même. Quand les employés sont satisfaits de ces conditions de travail, ils demeurent dans un état neutre ou état d'équilibre, la motivation n'est pas augmentée. Le mécontentement peut rompre l'équilibre mais en produisant des réactions négatives i.e., réduction de la production, etc. Les auteurs les ont appelé facteurs d'hygiène alors que les premiers facteurs sont reconnus comme facteurs de motivation. Les facteurs de motivation sont propres à l'individı et évoquent chez lui le besoin de trouver dans son occupation une source de croissanice: \& self actualization - ego - involvement ». Une telle révélation replace le syndrome de la motivation industrielle dans une perspective toute nouvelle.

$\mathrm{Par}$ suite des expérienres de Hawthorne et des études sur la dynamique des groupes de l'Université du Michigan (d'ailleurs de si nombreuses investigations n'ont pas tellement produit de résultats tangibles) l'industrie s'est lancée dans des programmes de relations humaines, de développement de la gérance, etc., tout ceci aux fins de produire des attitudes positives et d'augmenter ainsi la production. Le désenchantement fut cruel pour plusieurs, car les employés, quoique n'étant plus mécontents, n'en demeuraient pas moins assez neutres. En effet, tous ces programmes touchaient surtout aux facteurs d'hygiène et ignoraient le contenu du travail comme tel. Il n'est pas étonnant de constater qu'il a été difficile de trouver une relation statistique entre le moral et la performance.

A la suite de Hersey, Argyris, Taylor, Lawshe, etc., les auteurs ont démontré qu'il faut permettre autant que possible à l'individu de contrôler son travail, de faire quelque chose de personnel. D'ailleurs cette conception ne découle-telle pas directement de la nature humaine; que l'on se place du point de vue philosophique ou biologique, l'individualisme est un fait.

L'industrie moderne est une faillite du point de vue humain: d'un côté, la simplification du travail entreprise par la gérance appelle le conformisme en tuant toute initiative; de l'autre, le syndicat en prônant le concept sacré de l'ancienneté ignore trop souvent la compétence humaine.

Ce petit volume de cent cinquante pages est peut être la publication la plus importante à date sur la motivation de l'homme en tant qu'employé ou ouvrier. La survivance de la démocratie moderne ne peut commencer que par une réévaluation de nos valeurs sociales et individuelles. Cette transformation devra s'opérer d'abord dans le milieu du travail, puisque l'homme doit oeuvrer pendant une majeure partie de sa vie.

\section{C.-R. Giroux}

Englewood Cliffs, Occupational information, by C.L. Shartel, Prentice-Hall Inc., Englewood Cliffs, Third Edition, 1959.

L'orientation professionmelle tient maintenant une place importante dans notre système d'éducation. L'une des. 